\title{
COVID-19 Effects on Pregnancy, Neonates and Fertility
}

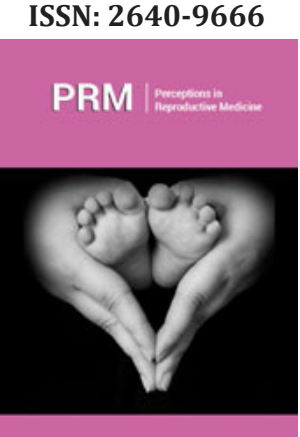

*Corresponding author: Ajit Kumar Saxena, Department of Pathology/ Laboratory Medicine, India

Submission: 眥 September 15, 2020

Published: 眥 October 06, 2020

Volume 4 - Issue 1

How to cite this article: Hani Raoul Khouzam. COVID-19 Effects on Pregnancy, Neonates and Fertility. Perception in Reproductive Medicine. 4(1). PRM. 000580. 2020.

DOI: 10.31031/PRM.2020.04.000580

Copyright@ Hani Raoul Khouzam, This article is distributed under the terms of the Creative Commons Attribution 4.0 International License, which permits unrestricted use and redistribution provided that the original author and source are credited.

\section{Hani Raoul Khouzam ${ }^{1,2 *}$}

${ }^{1}$ Staff Psychiatrist, Mental Health and psychiatry services, PTSD treatment program and General Mental Health Clinic, USA

${ }^{2}$ Clinical Professor of Psychiatry and Medicine, USA

\begin{abstract}
The Severe Acute Respiratory Syndrome Coronavirus 1 (SARS-CoV-1) which precipitated the COVID-19 pandemic, could adversely affect human reproductive health. This review summarizes the potential risks of COVID-19 on pregnancy, neonatal health and fertility as reported through clinical observation and recently published studies.
\end{abstract}

Keywords: COVID-19, pregnancy, neonates, fertility, coronavirus

\section{Introduction}

Coronavirus disease 2019 (COVID-19) is a novel type of highly contagious infectious disease caused by the severe acute respiratory syndrome coronavirus 2 (SARS-CoV-2).It has several medical complications that keep being observed and addressed on a daily basis as this global pandemic continues to progress worldwide. Some of the recently observed clinical findings have documented SARS-CoV-2 ability of affecting reproductive health and neonate's health. This review summarizes some of the recently described effects of COVID-19 infection on pregnant women, neonates and fertility. According to World Health Organization (WHO), pregnant women do not appear to be at higher risk of severe disease. Furthermore, WHO reports that currently there is no known difference between the clinical manifestations of COVID-19 in pregnant and non-pregnant women of reproductive age. Nevertheless, the available data on the exact effects of COVID-19 on fertility and pregnancy is scarce. Despite the increasing number of published studies on COVID-19 in pregnancy, there are insufficient rigorous and systematically collected data with regard to the severity of the disease or specific complications of COVID-19 in pregnant women, as well as vertical transmission, perinatal and neonatal complications and the capture of critical data is needed to better understand the effects of this infection on pregnant women and neonates [1].

\section{SARS-CoV-2 Effects on Pregnancy}

The main presenting symptoms of SARS-CoV-2 infection include fever, cough, fatigue, shortness of breath, sputum production, headache, and myalgias with some patients presenting with gastrointestinal symptoms [2]. Other patients may manifest anosmia which is an absence or decreased sense of smell or ageusia which is the loss or impairment of the sense of taste [3]. The severity of infection ranges from asymptomatic carriers, to mild flulike disease, to critical illness and death. Critically ill patients may experience respiratory failure, shock, or multiple organs failure [4]. Approximately $80 \%$ of infections are mild with flu-like symptoms, $15 \%-20 \%$ are severe, requiring hospitalization and supplemental oxygen, and $5 \%$ are critical and require mechanical ventilation [5]. The risk factors for severe illness include older age and the presence of co-occurring underlying medical conditions such as cardiovascular disease, diabetes, chronic respiratory disease, hypertension, and cancer [6]. Up to $3 \%$ of SARS-CoV-2 infections have been associated with increase mortality in individuals older than 60 years of age or in those with underlying medical conditions ,however it is important to recognize that death to COVID-19 can occur at any age including younger patients, and women of reproductive age who could be initially asymptomatic carriers of 
the virus [3]. Based on limited reports and the available data from other respiratory pathogens, it is unknown whether pregnant women with COVID-19 will experience more severe illness than nonpregnant adults. COVID-19 infection during pregnancy is not generally associated with an increased risk of spontaneous abortion and spontaneous preterm birth [7]. In some reports, SARS$\mathrm{CoV}-2$ was found to increase the risk for pregnancy complications especially in the third trimester including acute respiratory distress syndrome, disseminated intravascular coagulopathy, renal failure, secondary bacterial pneumonia, and sepsis [8]. To prevent these possible complications, the delivery should optimally be performed in health care facilities with close maternal and fetal monitoring. The timely delivery in women with COVID-19 critical status is not usually associated with increased risk of premature birth or asphyxia of the newborn and could improve the treatment and rehabilitation of maternal pneumonia [9].

Pregnant women with comorbidities such as obesity are likely at increased risk for severe illness consistent with the general population with similar comorbidities. However, given that pregnancy itself is now identified as a risk factor for certain outcomes, the magnitude of further increase from such comorbidities will need to be further delineated. Clinicians are urged to provide educational instructions to pregnant women and those contemplating pregnancy about the potential risk for severe illness from COVID-19, and measures to prevent infection with SARS-CoV-2 should be emphasized for pregnant women and their families. Pregnant individuals are encouraged to take all available precautions to optimize health and avoid exposure to COVID-19. These educational efforts could be provided via phone or telehealth sessions before scheduled appointments to allow clinics to appropriately prepare and optimize care coordination needs.

\section{SARS-CoV-2 Effects on Neonates}

There seem to be a low frequency of spontaneous preterm birth and general favorable immediate neonatal outcome in pregnant women with COVID-19 and the rate of vertical or peripartum transmission of SARS-CoV-2 is considered to be very low and not yet detected for cesarean delivery [10]. Although vertical transmission of SARS-CoV-2 was not detected, the proportion of neonatal bacterial pneumonia was higher than other neonatal diseases in newborns [11]. Although the majority of mothers do not develop major complications, severe maternal morbidity as a result of COVID-19 and perinatal deaths were reported suggesting that vertical transmission of the COVID-19 could not be ruled out because maternal infection can cause serious problems such as preterm labor and fetal distress [12]. Based on these observations the careful monitoring of pregnancies with COVID-19 and measures to prevent neonatal infection should be implemented [13]. Since early and close contact between the mother and neonate has many well-established physical, emotional and psychological benefits including increased success with breastfeeding, facilitation of mother-infant bonding, and promotion of family-centered care.
Although long-term effects are not yet fully identified, available data suggest that there is no difference in risk of SARS-CoV-2 infection to the neonate whether a neonate is cared for separately or remains in the mother's presence .The decision of whether to keep individuals with known or suspected SARS-CoV-2 infection and their neonates together or separate after birth should include a process of shared decision-making with the mother, the family, and the clinical team. This issue should be raised during prenatal care and continue through the intrapartum period. Healthcare providers should respect maternal autonomy in the medical decision-making process without any coercion, and facilities should implement policies that protect mothers' informed decision [14].

\section{SARS-CoV-2 Effects on Fertility}

Since SARS-CoV-2 has been identified in the semen of infected patients, it raises the possibility that testicular damage and subsequent infertility may result following COVID-19 infection, and also the possibility of sexual transmission, however until detailed physiological and pathological examinations of the male reproductive systems of COVID-19 patients after their recovery are conducted, the long-term impacts of SARS-CoV-2 on male reproductive function, including its potential effects on fertility and testicular endocrine functions is not definitely known at this time [15]. Available data suggest that SARS-CoV-2 infection is unlikely to have long-term effects on male and female reproductive function. Although these findings are not definitive, they imply that procedures in which oocytes are collected and fertilized in vitro are associated with very little risk of viral transmission from gametes to embryos and may indeed have the potential to minimize exposure of susceptible reproductive cell types to infection in comparison with natural conception [16]. The effect of COVID-19 on male reproductive function was evaluated by studying the male sex hormones namely testosterone ( $\mathrm{T}$ ), luteinizing hormone ( $\mathrm{LH}$ ) and follicle-stimulating hormone (FSH) It appears that $\mathrm{T}$ level is not affected by the infection, however the ratio of FSH to LH is significantly decreased [17]. However, the results of this study should be followed by a more direct analysis of the seminal fluid of COVID-19 patients to evaluate the effect-if any-on sperm count, volume, morphology or motility. Since many viral infections could causes orchitis in addition to other complications on male fertility, it is important to monitor fertility in males recovering from SARS$\mathrm{CoV}-2$. Although some reports have also indicated a decreased sperm concentration and motility for 72-90 days following COVID-19 infection; it is unclear whether SARS-CoV-2 adversely affects female gametogenesis [18]. In summary, currently, it does not appear that COVID-19 significantly impact fertility or conception in women, however its long-term impact on men's fertility is still undetermined.

\section{Conclusion}

In this brief review the impact of COVID-19 on pregnancy, neonates and fertility was summarized based on currently available 
research and clinical findings. Scientists, researchers and clinicians are conducting ongoing research and daily clinical observation in this important area of people livelihood. In the meantime, it is of paramount importance to implement policies and updated guidelines to monitor and prevent the spread of this novel and multifaced highly infectious and contagious novel virus.

\section{References}

1. Juan J, Gil MM, Rong Z, Zhang Y, Yang H, Poon LC, et al. (2020) Effect of coronavirus disease 2019 (COVID-19) on maternal, perinatal and neonatal outcome: systematic review. Ultrasound Obstet Gynecol 56(1): 15-27.

2. Pan L, Mu M, Yang P, Sun Yu, Runsheng W, et al. (2020) Clinical characteristics of COVID-19 patients with digestive symptoms in Hubei, China: A descriptive, cross-sectional, multicenter study. Am J Gastroenterol 115: 766-773.

3. Mao R, Qiu Y, He JS, Jin Yu Tan, Hua Li X, et al. (2020) Manifestations and prognosis of gastrointestinal and liver involvement in patients with COVID-19: A systematic review and meta-analysis. Lancet Gastroenterol Hepatol 5(7): 667-678.

4. Segars J, Katler Q, McQueen DB, Kotlyar A, Glenn T, et al. (2020) Prior and novel coronaviruses, Coronavirus Disease 2019 (COVID-19), and human reproduction: What is known? Fertil Steril 113(6): 1140-1149.

5. World Health Organization (2020) Coronavirus disease 2019 (Covid-19) situation report 46.

6. CDC (2020) Interim clinical guidance for management of patients with confirmed coronavirus disease (COVID-19).

7. Yan J, Guo J, Fan C, Juan J, Xuechen Yu, et al. (2020) Coronavirus disease 2019 in pregnant women: A report based on 116 cases. Am J Obstet Gynecol 223(1): 111e1-111e14.
8. Rasmussen SA, Smulian JC, Lednicky JA, Tony SW, Denise JJ, et al. (2020) Coronavirus disease 2019 (COVID-19) and pregnancy: what obstetricians need to know. Am J Obstet Gynecol 222(5): 415-426.

9. Lapinsky SE (2017) Management of acute respiratory failure in pregnancy. Semin Respir Crit Care Med 38(2): 201-207.

10. Parazzini F, Bortolus R, Mauri PA, Favilli A, Gerli S, et al. (2020) Delivery in pregnant women infected with SARS-CoV-2: A fast review. Int J Gynaecol Obstet 150(1): 41-46.

11. Zhang L, Dong L, Lei Ming L, Wei M, Jun Li, et al. (2020) Severe acute respiratory syndrome coronavirus 2 (SARS-CoV-2) infection during late pregnancy: A report of 18 patients from Wuhan, China. BMC Pregnancy Childbirth 20: 394.

12. Dana PM, Kolahdooz F, Sadoughi F, Moazzami B, Chaichian S, et al. (2020) COVID-19 and pregnancy: A review of current knowledge. Infezioni in Medicina 28(Suppl1): 46-51.

13. Zaigham M, Andersson O (2020) Maternal and perinatal outcomes with COVID-19: A systematic review of 108 pregnancies. Acta Obstet Gynecol Scand 99(7): 823-829.

14. Baecher Lind L, Fleming AC, Bhargava R, Susan CM, Elise EN, et al. (2020) Medical education and safety as co-priorities in the coronavirus disease 2019 (COVID-19) Era: We Can Do Both.

15. Fraietta R, Pasqualotto FF, Roque M, Taitson PF (2020) SARS-COV-2 and male reproductive health. JBRA Assist Reprod 24(3): 347-350.

16. Stanley KE, Thomas E, Leaver M, Wells D (2020) Coronavirus disease-19 and fertility: Viral host entry protein expression in male and female reproductive tissues. Fertil Steril 114(1): 33-43.

17. Ma L, Xie W, Li D, Shi L, Mao Y, et al. (2020) Effect of SARS-CoV-2 infection upon male gonadal function: A single center-based study. MedRxiv.

18. Li D, Jin M, Bao P, Zhao W, Zhang S (2020) Clinical characteristics and results of semen tests among men with coronavirus disease 2019. JAMA Netw Open 3(5): e208292. 